suprapubic region, including the detached organs. The vessels of the penis were now secured separately, and any other vessels which bled were also tied. The result of the operation was a very large wound, the edges of which could not of course be brought together, but a few stitches through the perineal portion slightly diminished this part of the wound. The whole wound was freely sponged with De Morgan's solution of the chloride of zinc, and lint soaked in carbolic oil was applied over it.

Oct. 23rd.-The patient's progress since the operation has been most satisfactory. His temperature is now normal, and his general health is improving. The wound continues to be dressed with carbolic oil, which is renewed every two hours.

Nov. 1st. - The general condition and wound are still progressing favourably; the latter is steadily contracting. The orifice of the urethra remains free, and the patient passes urive easily and without pain.

Dec. 30th.-To-day the patient was dismissed cured, and much improved in health, his emaciation having quite disappeared. The wound is now healed, and the orifice of the urethra remains open, so that there is no difficulty in passing urine. It is also noted that the glandular enlargement has much diminished.

The patient promised to report if any return of the disease took place; but as up to this date (December, 1874) he has not been heard of, it is understood that he remains well.

Remarks.-This case is encouraging in connexion with the treatment of epithelial cancer, and proves that a serious operation may be successfully performed for its relief, provided that the entire disease can be removed, and the patient's condition admits of such a proceeding. The duration of the disease and the marked improvement of the patient's health after its removal point very ảecidedly to the local nature of the cancer in this case. The prevention of hæmorrhage during the operation was an important question in this case, for it is well known that the vessels connected with the genital organs bleed freely when divided. The method adopted in the present instance for restraining the hæmorrhage was most successful, and the operation was completed with a comparatively slight loss of blood-a circumstance which no doubt assisted in promoting the excellent recovery of the patient.

Edinburgh.

\section{NOTES ON THE}

\section{LOCAL USE OF LIQ. FERRI PERCHLORIDI FORTIOR IN CANCEROUS ULCERA- TIONS OF THE UTERUS.}

By CHARLES J. GIBB, M.D., CONSULTING SURGEON TO THE NEWCASTIE INFIRMARY.

Cancerous diseases of the uterus have generally progressed so far before they come under professional observation as to pass as incurable from one medical man to an. other; and it thus happens that I see a large number in my consulting rooms. It is rare to find the disease so superficial and purely epithelial in character, or, if interstitial, so confined to the mouth and neck of the uterus, that the diagnosis can be made with such certainty as to justify the surgeon in excising it; and, in the advanced stages, the foul discharges make the case so loathsome that, in spite of the keenest feelings of pity, the surgeon is inclined to view the case as utterly hopeless, and to leave all nianual treatment to the nurse. When we remember how nuch relief can be given to the worst symptoms of the most incurable cases of external cancers by operation, or other measures calculated to remove fungoid or sloughing surfaces or masses of the disease; how pain can thus be relieved, bleeding prevented, foul discharges moderated or made less disgusting in character,-it is not surprising that surgeons should strive to give similar relief to the worst cases of cancer of the womb, and be more or less successful in their efforts. From time to time I have tried the various local applications I have seen recommended, have made use of many caustics, been taught by painful experience that caustic potash or caustic potash and lime are unmanageable and too dangerous to the surrounding parts to be used with the freedom requisite to be of service, that the actual cautery is too fear-inspiring, that nitric acid and acid nitrate of mercury (which have to be used with great care) are inefficient, and that lunar caustic and powders or points of alum are useless as caustics.

About two years ago I had occasion to dilate the uterus in a very obstinate case of menorrhagia, proceeding from large vascular granulations in the cavity of the enlarged organ. The strongest Pharmacopoial solution of the perchloride of iron (being, in fact, iron dissolved in pure acid) was injected into the open uterine cavity, and a piece of sponge soaked in the solution left there for twenty-four hours. This treatment was perfectly successful; and I viewed with surprise the efficient way in which the soft and vascular growths were destroyed, whilst the normal structures of the uterus and vagina were but little inconvenienced, and certainly not in the least eroded. Since that time I have been accustomed to use this solution in many cases of cancer of the uterus; and I jotted down the histories of the following four cases, being cases of cancer in which $I$ used it in my consulting rooms during one week.

CASE 1. - Mrs. B- aged thirty-six; a shopkeeper; married five years; no children. Was a strong, powerful woman until fourteen months ago, when she was seized with menorrhagia, followed by the foul sanious discharges and other symptoms of cancerous ulceration. She came to my offices six weeks ago, anæmic to the last degree from almost constant bomorrhage and putrid discharge. On examination, the mouth and neck of the uterus were found destroyed, and their place occupied by a large, deep, sloughing, cancerous sore. The body of the uterus felt greatly enlarged and as hard as a cricket-ball, whilst the vagina was quite free from disease. Profuse hæmorrhage attended the examination. The sore was filled with cotton-wool soaked in the solution of the perchloride, and the vagina stuffed with tow. She came from a distance by rail, and was ordered to remove with her fingers, or allow the lady who accompanied her to withdraw, the stuffing of tow next morning, and trust to the injections of zinc and alum to wash away the cotton-wool ; and appropriate blood-making and aperient medicines were prescribed. On her second visit she expressed herself as much stronger, very little bleeding having taken place during the week. The application of the perchloride was repeated, and I did not see her for the next ten days, in consequence of a severe attack of pain compelling her to remain in bed. The sore was much healthier in appearance, there had been very little bleeding, and the discharge had become scanty and semi-purulent in appearance, without any of the old foul, putrid smell. She was, however, very feeble, and made the journey with great difficulty. Instead of placing the cotton-wool soaked in the perchloride over the sore, I elevated her breech, half filled the vagina with the solution for a couple of minutes, then sucked it up with a syringe, and left a plug of tow in the vagina, to be removed next day. The improvement was marked on her fourth visit; and on her sixth I found there had only been a little bleeding once during the week, whilst forcing away a very costive motion. The ulcer was perceptibly smaller and free from slough, the circumference of the vagina around it having contracted considerably. A small quantity of yellow, oil-like pus lay in the vagina, but the examination still caused the ulcer to bleed slightly. The ulcer was again bathed with the strong perchloride, and she returned home, expressing herself as twice as strong as when she first called upon me, and very much relieved from the local misery.

CASE 2.-Mrs. R-, aged thirty-six, an innkeeper, with several children, the last seven years old. Began to have menorrhagia and the ordinary symptoms of cancer of the womb nearly a year ago. Commenced attending my offices four montbs ago. Was found to have a large, soft, bleeding epithelioma, covering the swollen and apparently destroyed vaginal part of the uterus, and extending along the front wall of the vagina to within an inch and a half of the orifice of the urethra. She came in a cab, and her linen was drenched with blood. She was excessively anæmic from daily hæmorrhage, and was evidently in the last stage of the disease, the sore bleeding on the least examination. A large piece of cotton wool was soaked in the perchloride, placed over the disease, and the vagina stuffed with tow. 
On her second visit, a week afterwards, the hæmorrhage had almost ceased, the sanious putrid discharge was much lessened, and, although there was little change to be seen in the appearance of the sore, the finger felt it to be less fungoid and pultaceous in consistence, and less blood fol lowed the examination. I may here remark that the bleeding fungoid sores were so extensive as to make it utterly impossible to use any speculum, and the oiled fingers of myself and assistant had to be used to open the ragina and make the necessary examinations and applications. The same examination was made weekly during the nine succeeding visits, by which time she had regained much of her lost strength, walking a considerable distance to $\mathrm{my}$ rooms, and experiencing but little pain or inconvenience except in passing urine and fæees. The hæmorrhage had ceased after the third application; the foul sanious discharge had given place to a scanty oil-like purulent matter without smell; the fungoid vaginal sore was healed, or rather converted into a thick nodulated gristly cicatricial substance, covered with a smooth membrane, whilst the deepest partthat corresponding to the uterus-alone presented a chinklike ulcerated surface. It was difficult to see or to reach the deepest part on account of the remarkable contraction that had taken place in the calibre of the vagina, which, from being very capacious and soft, admitting several fingers, had become changed to a rigid tube, that would scarcely allow more than one finger to pass along the upper part of the canal. At this period some of her children became ill of fever, and she did not visit me for six weeks, having gone through much fatigue in the nursing of them. On examining her this week, on her return, I found she was again suffering from a recurrence of the bæmorrhage, consequent, as she asserted, upon the menstrual flow, and I also found that the disease had again opened out the deepest part of the cicatrix, and formed an irregular foul ulcer nearly the size of a crown-piece. The front part of the cicatrix remained in much the same condition; I covered the sore with wool soaked in the perchloride, and warned her against such negligent attendance in future.

CASE 3.-Mrs. B-, aged forty-six, a stout, healthylooking lady from the country, the mother of several children, called upon me about a year ago, having slight discharge and bleedings in the intervals between ber monthly periods, as well as excessive monthly flow. Her other symptoms were so slight that her husband (who was also a patient) had difficulty in persuading her to see me. The mouth and neck of the uterus were found red and raw. looking, bleeding on the slightest touch, also hard and considerably enlarged, and there was an ulcerated crack at the orifice of the organ. She attended my rooms once a week for about two months. Lunar caustic was applied at first, but, producing little change, the perchloride was used, for a few times with such good effect that, considering herself quite well, she did not return to see me until now, when she tells me the bleedings have returned lately, and she feels a heavy, uncomfortable weight in the uterine region. On examination the whole organ is found greatly enlarged and very hard, being evidently infiltrated with scirrhus disease. The old ulcer is again open, bleeds freely on examination, and there is considerable discharge. The perchloride was applied.

CASE 4.-Mrs. F_, aged sixty-nine, from the sea coast, consulted me about eight years ago, for a discharge, occasional menorrbaria, and the uncomfortable bearing down and other symptoms indicative of uterine mischief. The uterus was found atrophied, and the os and neck almost destroyed by a chronic cancroid ulcer, in appearance like to lupus, the whole case reminding me strongly of a similar condition of the breast, which I have of ten seen in old people to continue in a chronic state for years. Potassa fusa and other caustics and injections were used for more than a year, the result being the total destruction of the vaginal part of the uterus, and the formation of a deep sulcus there, which, after remaining healed for some months, again opened out, and has ever since compelled her to attend my rooms two or three times a year for a month or two together. A slight bloody discharge is the only symptiom that now tells her the cicatrix has given way, and of this discharge she has the greatest dread. Lunar caustic or nitric acid, with the use of injections, were generally successful after a time in healing the sore, or rather crack, in the cicatrix. I am now accustomed, however, to fill the wound with a small piece of wool dipped in the perchloride and retaining it there, for the first day, by a plug of tow in the vagina, and find it frequently, in spite of the use of injections, to stick in the place until her next visit, a week afterwards, when [ remove it. I find the perchloride to be by far the most effectual means of healing the scar when it cracks and bleeds, and on this occasion $I$ made the usual application.

In reviewing the above cases, I have to remark that since the first case was committed to paper the patient has visited me twice, and on each occasion has reported large losses of blood, although on examination the sore looks clean and the discharge remains a thick brownish pus quite devoid of smell. What remains of the uterus is infiltrated with cancer, and although the treatment has greatly relieved the most distressing symptoms, it is evident that a fatal termination cannot be long delayed. Indeed, I have seen that where the form of cancer is hard and embraces the whole of the uterus, the destruction of the enlarged organ of ten takes place by a rapid disintegration, attended with alarming gushes of blood, and all that can be done only lengthens the downward path and makes it less foul and painful. It is different with that class of cases of which the second is a good example. Here there was as pure an epithelioma as is met with in the uterus, spreading over the adjoining surfaces of the vagina, and not infiltrating or running deeply into their structures, but throwing out surfacewards exuberant vascular fungoid granulations, quickly renewed as the older parts sloughed away. Into this soft albuminoid structure the acid solution of iron sinks, destroys, and disinfects, and, strong as it is, only hardens and tans, but does not in the least destroy the healthy mucous membrane of the ragina. A few applications of the cotton-wool soaked in the solution suffice to clear away the diseased growth. Reparative efforts are then made by the comparatively healthy structures underneath, and the further applications appear to basten cicatrisation as well as eradicate the remains of the disease. In one case very similar to the second case, the lady has now been quite well for some months, and the resulting cicatrix, when she ceased to attend upon me, had so narrowed the upper third of the vagina that the finger could not pass along it, and was so thick, hard, and tuberculated that I could not resist coming to the conclusion that in the process of cure a large amount of albuminoid matter had exuded into and become solidified in the structures surrounding the disease. Whether the thickening and solidification of the cicatrix and immediately adjoining parts is what naturally takes place in this region when epithelial cancer is destroyed by other caustics, I have no experience to know; possibly it may partially result from the action of the solution left for so long a time on the highly vascular structures supporting the diseased growth-structures that have been so long accustomed to throw into the diseased mass the large supply of albuminoid food necessary for its support. In another case of apparent cure, where the epithelial disease was confined to the uterine structure, I did not observe this condition of the cicatrix; and it may be that the presence of so much connective tissue in the vaginal wall is the cause, as well as the seat, of this peculiar formation, reminding me, as it does, of a bad case of keloid growth in the skin, or the hard irregular cicatrix of a bad burn. The course of cancer in the womb is sometimes so rapid from the quick disorganisation and sloughing of the uterus, and the sufferings of the patient are so great, that it is impossible, in a dispensary practice like mine, to see such cases as of ten as necessary, and to follow them out satisfactorily, or to persuade a sensitive woman to have repeated applications of an agent which does not give im. mediate or permanent relief. In such cases, indeed, no remedies can be ol much avail. It is where the disease is purely epithelial, or chronic or rodent in character, and confined to the surface, that the treatment I have described does most good, and, as I have said, appears sometimes to cure even bad cases. The application of the perchloride rarely causes pain. On four or five occasions a patient, on returning home, has been confined to bed for a few days, and, in one of the successful cases, for upwards of a fortnight, in consequence of a severe colic-like pain in the region of the uterus, lower abdomen, and back. I am inelined to think that the wool had been over-saturated in the perchloride in such cases, as it has occasionally bappened that 
the solution has flowed from the vagina over the vulva after the patient has left my offices, and those parts been blistered and painfully excoriated as a consequence. On this account I am now very careful to wash away with a syringe all discharges from the surface of the cancer, to raise the breech of the patient to prevent any overflow of the solution over the vulva, and, after applying the perchloride, to suck up with a sponge from out of the bottom of the vagina any superabundant solution which a slight pressure on the saturated wool over the sore may cause to flow out; after that, to retain the wool in its place by a loose plug of tow in the vagina, and, lastly, dry and oil the vulva before the patient rises from the couch.

I have always used the strongest Pharmacopoial solution undiluted, as I have only used it to secure a caustic action. At first $I$ applied it on pieces of sponge or lint, but finally found cotton-wool to answer best, as it sucks up any quantity that may be required, parts with it readily, and can be moulded into any form so as to fill a cavity, or cover over and adhere to any growth. It has happened occasionally that I have found the cotton-wool still adherent over the sore a week or more after its application, and, when removed, it is always a black or chocolate-coloured mass, frequently quite solid from the quantity of blood or albuminous matter absorbed into its meshes and clotted thereinindeed, one patient gravely told me she had passed a solid brown egg a few days after one of her visits. No doubt it was the hardened wool, although she declared she bad cleared out the vagina the day after her visit.

I have kept no record of the cases I have treated with the perchloride; but as I have generally had six or eight under treatment at one time, I must have used it in twenty or thirty cases, and its beneficial influence has been so marked that I would strongly recommend its trial in suitable cases.

\section{PARASITIC VEGETABLE FUNGI, AND THE DISEASES INDUCED BY THEM.}

DISCUSSED FROM SPECIAL STANDPOINTS.

By STAFF-SURGEON THOMAS COLAN, M.D., R.N.

(Communicated by the Director-Generat of The Medical Department OF THE NAVY.)

(Concluded from page 757.)

3. How Parasitic Vegetable Fungi are communicated to and enter the substances they are found in.

Fungr develop by means of spores or seeds, throwing out a mycelium, as the spawn of mushrooms, and this mycelium grows into a cellular plant; the whole structure of a fungus might be called a thallus, save the reproductive organs which are found in or upon it. Such fungi can enter living bodies, either wholly or in spores, in four ways-viz., by means of respiration, deglutition, absorption through the skin or mucous membrane, and by inoculation.

1st. By respiration. Spores flying about in the air are conveyed to the mouth, nostrils, and lungs, or to any kind of breathing apparatus. Sporules of the Botrytis brasianæ, emanating from a dead or unhealthy silkworm, are carried in the air into the breathing pores which open in to the tracheal system of another worm, get to the fatty tissue beneath the skin, and developing there, destroy it at a time when the insect is most in want of nutriment. Individuals of a species of Polistes (the representative of our wasp) fly about in the West Indies with plants the length of themselves projecting from some parts of their surfaces, which plants were probably introduced at the breathing pores at their sides. They kill the insects, and in all likelihood flourish better on them dead than living. Again, different species of the genus Sphœria grow in bodies of certain caterpillars in New Zealand, Australia, and China. The insects get full of the fungus, and, when dried, are almost as solid as wood, and present the appearance of twigs with long slender stalks, formed by projections of the fungus. If such be the case here, it is probably so in other cases. For instance, that the spores of Oidium albicans are drawn in to the nostrils and mouth of human beings, causing aphtbæ and diphtheria, and in some cases enter the blood, and getting to the remote mucous membrane, flourish there.

Vegetables which respire in air doubtless receive in the inspired gas the spores which generate disease.

2nd. By deglntition. We know that a young cestoid worm, when swallowed with water by human beings and swine, perforates the stomach, from whence by the blood it is carried to some capillary vessel, the coats of which it gets through, and lodges in muscular, glandular, or brain tissue, where it develops. By analogy, we may argue that the sporules of a parasitic vegetable fungus can act in a similar manner. Man eats wheat, very few grains of which, according to Dr. Marshall, are not infected near their points with one or two fungous sporules entangled in the minute hairs; he swallows fruits, as the pine, on which the Ecidium cancellatum is found; he partakes of leguminous vegetables, as the pea, bean, and pulse, and of unbelliferous plants, as the celery and parsley, all of which are liable to attacks of mildew fungus; he eats potatoes and turnips, on which the Botrytis exists; he partakes of fish, on the gills, scales, shells, and other hard portions of which parasitic vegetable fungi appear; he eats mouldy bread, fruits, and cheese,* and of animals infected with fungi.

As regards vegetables, we have reason to believe, from Sée's experiments, that the spores of the Uridinew are taken up in the roots of the plant, and find their way with the sap. to the top, the proper place for their growth.

3rd. By absorption through the skin or mucous membrane. Women in India affected with Tinea circinata, caused by the fungus Tricophyton tonsurans, have been known to communicate such disease to men.

Devergie, von Barensprung, and others have proved that parasitic diseases may be transmitted from horses, oxen, and other animals to man; and Dr. Fox relates an instance of a white cat which contracted the mange from Tinea tonsurans, with which the children of the family to which it belonged were affected. It may be that vegetables infect other vegetables through their coats, or vegetables com. municate spores to animal substances.

4th. By inoculation. Fungi can be conveyed to the blood by inoculation. Kobner by this means, in 1864, caused Tinea circinata to appear in his own arm. A vegetable injured on its surface might possibly become affected in this manner by the fungi of vegetables or animals. Inoculation can also be effected through the mucous membrane. The spores having entered living substance, find suitable habitats, and exhibit there the stages of growth or varieties of the species to which they belong; and we name that exhibition a particular disease. The virulence of the latter and the luxuriance of the former will depend on circumstances-temperature, for instance. It has been thought that fungi abound most in cold regions, but $I$ am disposed to think this belief has resulted from want of more perfect observation, at least as regards parasitic vegetable fungi. I have seen more Tinea circinata in one commission, whilst serving in H.M.S. Malabar in the Red Sea and India, than during over seventeen years I have served elsewhere, and in that ship suffered myself from the complaint. Is the ordinary vegetable productions in the Tropics far surpass in size and luxuriance those of colder regions, so it is likely the same is the case with parasitic vegetable fungi. The great heat and moisture, the greater amount (at times) of electricity in the air, the greater loss of alkalies, inducing an acid diathesis-a state generally favourable to parasitic fungous growth,-perhaps the greater a mount of pigment in coloured persons, and the abundance of odoriferous productions in their cutaneous system, together with the general siverve from the normal state of health of Europeans residing in warm climates, may all tend to the greater development of parasitic vegetable fungi, and aggravation of the diseases induced by them. Again, a bad or unhealthy state of body is necessary to the development of parasitic vegetable fungi, and the more unhealthy the constitution the more virulent will be the disease. "It is certain," says Dr. Aitken, "that wherever the normal chemical processes of nutrition are impaired, and the incessant changes between

* The blue mould of cheese (Aspergillus glaucus) is considered by epicures to give the cheese an agreeable flarour, and it is probable that it helps the digestion of other aliments. Again, a good portion of the stinking rust in flour is supposed not to render it unwholesome, at least when made into fermented bread. The fiour is largely used in the manufacture of gingerbread, where the treacle disguises the colour and faronr. 